\title{
Realistic parameters for dynamic simulation of composite cables using a damped Cosserat rod model
}

\author{
Dominik Jungkenn $^{1}$, Fabio Schneider-Jung ${ }^{1}$, Fredrik Andersson ${ }^{2}$ and Joachim Linn ${ }^{1}$ \\ ${ }^{1}$ Fraunhofer Institute for Industrial Mathematics ITWM \\ Department Mathematics for the Digital Factory (MDF) \\ Fraunhofer Platz 1, 67663 Kaiserslautern, Germany \\ (dominik.jungkenn, fabio.julian.schneider-jung, joachim.linn)@itwm.fraunhofer.de \\ ${ }^{2}$ Fraunhofer Chalmers Research Centre for Industrial Mathematics FCC \\ Department Geometry and Motion Planning \\ Chalmers Science Park, 41288 Gothenburg, Sweden \\ fredrik.andersson@fcc.chalmers.se
}

\begin{abstract}
Digital prototyping presents one of today's biggest chances in boosting efficiency of product development in automotive industry. Handling flexible parts, such as cables and hoses, is a big challenge in this context. The software IPS Cable Simulation addresses this topic and solves the problem for a wide field of applications. To obtain reliable simulation results, a basic set of parameters describing the effective mechanical properties of the flexible parts is an essential part of the model. The development of the MeSOMICS measurement machine represents a practical solution to this need for experimental data. Additional challenges are related to dynamic simulations of vehicles in operation mode. To solve these, we introduced the durability and dynamics module as an extension to the established software. This leads to an extended set of required parameters. In order to obtain these dynamic parameters, two different experimental setups have been realized. The experiments investigate damped torsional and bending oscillations, and yield parameters that can conveniently be treated as effective viscous properties within the framework of our software.
\end{abstract}

Keywords: cable simulation, dynamics, Cosserat rods, damping parameters, measurements.

\section{MOTIVATION AND STATE OF THE ART}

In today's automotive industry, digital validation at an early stage has become a standard procedure in product development. This includes the validation of flexible parts such as cables and hoses. The variety of electronics used in modern vehicles has made the cable system one of the central parts when it comes to safety and function of the product. So far the simulation based validation of cables and hoses using the IPS software family has focused on the basic functionality of the vehicles, namely the proper design of moving parts (such as doors, lids or the suspension linkage and steering) as well as the validation of production processes (assembly and disassembly by human workers as well as robotic applications). We aim to extend the field of application for our software by simulations of products in more dynamical operation, where inertial and damping effects need to be considered, and generalize our models and simulation methods correspondingly.

\subsection{Classical linear viscoelasticity}

Extending the range of applications from static or sequentially performed quasistatic equilibrium computations to fully dynamic simulations driven by transient structural excitations, we need to include viscoelastic constitutive properties in our structural model to account for internal damping effects, besides elastic structural response and inertia. Very common are one-dimensional rheological models for this purpose, as have been described e.g. in [1], and more recently by [2] with 


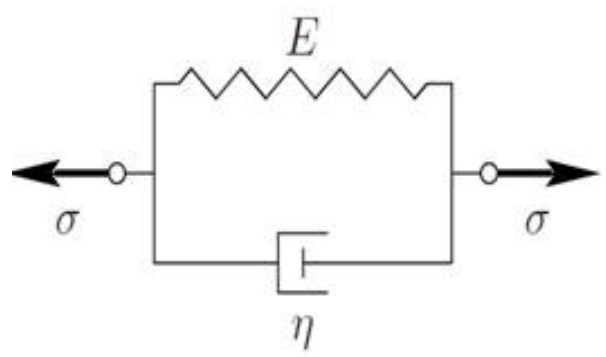

Figure 1: Schematic visualisation of a Kelvin-Voigt damping model

a focus on flexible multibody systems. These models usually consist of serial or parallel combinations of linear or nonlinear springs and dashpots, forming models of varying complexity. The Kelvin-Voigt (KV) type damping model, which we introduced in our previous work [3], is on the more simple part of the scale. It consists of an elastic spring-type and a viscous damper-type element connected in parallel (see Fig. 1). We refer to the conference paper [4] for basic numerical experiments exploring Cosserat rod dynamics with Kelvin-Voigt type viscous damping.

In the one dimensional case, the stress strain relation of the Kelvin-Voigt model reads:

$$
\sigma=E \varepsilon+\eta \dot{\varepsilon}
$$

Eqn. (1) with elastic modulus $\mathrm{E}$ and viscous damping $\eta$ results as an approximation of a more general rheological model of Generalized Maxwell (GM) type in the low frequency range, as briefly discussed in section 4.4 of [5]. In 3D solid mechanics, a similar constitutive model can be stated in tensorial form [6] following [7], as has been shown in [5] and more recently discussed by Bauchau and Nemani [2]. The latter paper focuses on Maxwell-type damping behavior of beams with rectangular cross-sections and non-negligible cross-sectional warping and is the most recent publication related to our research. The presented numerical experiments conclude in a general dissuasion against Kelvin-Voigt type modeling of viscoelastic beams. However, the use-cases presented by Bauchau and Nemani differ to a large extent from our field of application.

It turns out that in our application case the benefits of this simpler model outweigh the potential drawbacks of the model. In the so called Standard Linear Solid (SLS) model - this is defined [1] as a GM model with only one viscous Maxwell element, consisting of an elastic spring and a viscous damper in row, parallel to the elastic spring, equivalent to a one term Prony series as well as in GM models of more complex type, stiffness and damping parameters are inevitably coupled as real and imaginary parts of a complex response function (see [14] Ch. XII §123). While this reflects the built-in causality of the material response, which is certainly a not only desired, but mandatory property for a proper material model, the hard wired coupling of elastic stiffness and viscous damping makes parameter identification for GM models a tedious task. Differently, the two parameters of the KV model can be adjusted independently, which turns out to be a favourable feature if one tries to match the effective, cross sectional response of a complicated composite structure as a cable (see Fig. 2). Trying to achieve the same with the 3 parameters of SLS model,
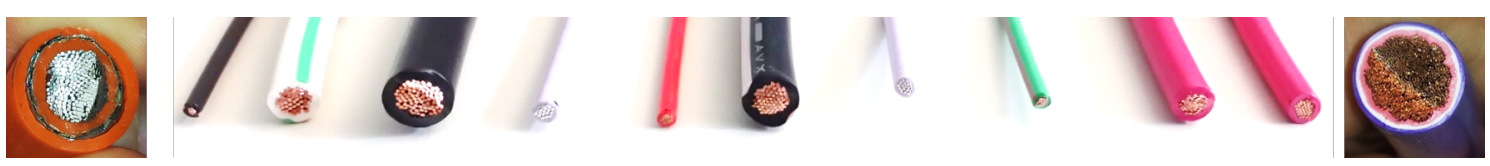

Figure 2: A collection of composite cables with complex cross sectional structure

our experience shows that one encounters substantial difficulties.

Therefore we still regard the KV approach to low frequency viscoelastic response of composite cable structures as the prefered choice. This paper aims to further substantiate that claim by applying physical experiments. 


\subsection{Viscoelastic rods}

Most works on rods with viscoelastic properties deal with linear Euler-Bernoulli or Timoshenko beam models. In contrast, viscous damping models for geometrically nonlinear beams or rods are rarely found in literature. Notably, Antman [8] introduced a damping model of the form

$$
\boldsymbol{F}=\hat{\mathbb{C}}_{F} \cdot\left(\boldsymbol{\Gamma}-\boldsymbol{\Gamma}_{0}\right)+\hat{\mathbb{V}}_{F} \cdot \dot{\boldsymbol{\Gamma}}, \quad \boldsymbol{M}=\hat{\mathbb{C}}_{M} \cdot\left(\boldsymbol{K}-\boldsymbol{K}_{0}\right)+\hat{\mathbb{V}}_{M} \cdot \dot{\boldsymbol{K}}
$$

with the cross section integrated viscous response given in the effective constitutive equations (2) for the material sectional forces and moments characterized by the diagonal matrices

$$
\hat{\mathbb{V}}_{F}=\operatorname{diag}\left(\gamma_{S 1}, \gamma_{S 2}, \gamma_{E}\right), \quad \hat{\mathbb{V}}_{M}=\operatorname{diag}\left(\gamma_{B 1}, \gamma_{B 2}, \gamma_{T}\right)
$$

with positive, but otherwise undetermined parameters. Antman's approach was primarily motivated as an artificial, regularizing viscous modeling element introduced to suppress the formation of shock waves in the hyperbolic dynamics of elastic Cosserat rods. For a brief review of other related work, we refer to [5], as well as to the more recent work of Bauchau et al. [2].

\section{SHORT OVERVIEW OF THE VISCOELASTIC COSSERAT ROD MODEL}

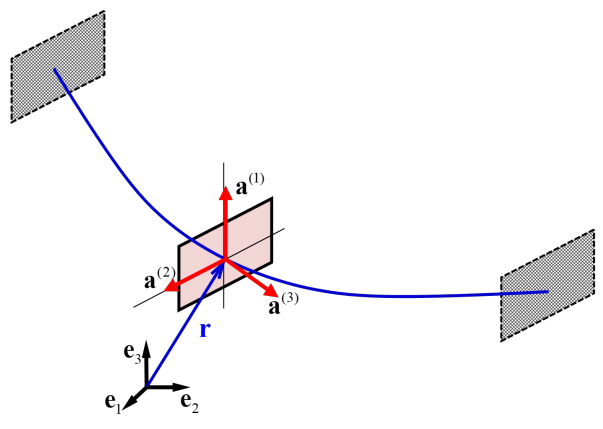

Figure 3: Schematic sketch of the centerline curve and moving frame defining a Cosserat rod.
The geometrically exact theory of Cosserat rods has been introduced by [9] and [10]. Following their work, our Cosserat rod is defined by its centerline curve $\boldsymbol{r}(s, t)=r_{k}(s, t) \boldsymbol{e}_{k}$, with cartesian component functions $r_{k}(s, t)$ w.r.t. the fixed global orthonormal frame $\left\{\boldsymbol{e}_{1}, \boldsymbol{e}_{2}, \boldsymbol{e}_{3}\right\}$ of Euclidian space, and the "moving frame" $\hat{\boldsymbol{R}}(s, t)=\boldsymbol{a}^{(k)}(s, t) \otimes \boldsymbol{e}_{k} \in S O(3)$ of orthonormal directors, booth smooth functions of the curve parameter $s$ and the time $t$. The local cross sections with normals $\boldsymbol{a}^{(3)}$ along the rod are spanned by the pair of directors $\left\{\boldsymbol{a}^{(1)}, \boldsymbol{a}^{(2)}\right\}$, as sketched in Fig. 3.

\subsection{Material strain measures}

The material strain measures associated to the configuration variables are given by the components $\Gamma_{k}=\boldsymbol{a}^{(k)} \cdot \partial_{s} \boldsymbol{r}$ of the tangent vector in the local frame (i.e.: $\boldsymbol{\Gamma}=\hat{\boldsymbol{R}}^{T} \cdot \partial_{s} \boldsymbol{r}=\Gamma_{k} \boldsymbol{e}_{k}$ ), with $\Gamma_{1}, \Gamma_{2}$ measuring transverse shear deformation and $\Gamma_{3}$ measuring extensional dilatation, and the material Darboux vector $\boldsymbol{K}=\hat{\boldsymbol{R}}^{T} \cdot \boldsymbol{\kappa}=K_{k} \boldsymbol{e}_{k}$, obtained from its spatial counterpart $\boldsymbol{\kappa}=K_{k} \boldsymbol{a}^{(k)}$ governing the Frénet equations $\partial_{s} \boldsymbol{a}^{(k)}=\boldsymbol{\kappa} \times \boldsymbol{a}^{(k)}$ of the frame directors, with $K_{1}, K_{2}$ measuring bending curvature w.r.t. the director axes $\left\{\boldsymbol{a}^{(1)}, \boldsymbol{a}^{(2)}\right\}$, and $K_{3}$ measuring torsional twist around the cross section normal. In general, the reference configuration of the rod, given by its centerline $\boldsymbol{r}_{0}(s)$ and frame $\hat{\boldsymbol{R}}_{0}(s)=\boldsymbol{a}_{0}^{(k)}(s) \otimes \boldsymbol{e}_{k}$, may have non-zero curvature and twist (i.e.: $\boldsymbol{K}_{0} \neq 0$ ). However we may assume zero initial shear $\left(\Gamma_{01}=\Gamma_{02}=0\right)$, such that all cross sections of the reference configuration are orthogonal to the centerline tangent vector, which coincides with the cross section normal (i.e.: $\left.\partial_{s} \boldsymbol{r}_{0}=\boldsymbol{a}_{0}^{(3)} \Rightarrow \Gamma_{03}=1\right)$ if we choose the arc-length $s$ of the reference centerline as curve parameter. 


\subsection{Dynamic equilibrium equations}

The constitutive equations (2) - or more general ones of viscoelastic type (see Ch. 8.2 in [11]) are required to close the system of dynamic equilibrium equations

$$
\begin{gathered}
\partial_{s} \boldsymbol{f}+\boldsymbol{f}_{\text {ext }}=\left(\rho_{0} A\right) \partial_{t}^{2} \boldsymbol{r} \\
\partial_{s} \boldsymbol{m}+\partial_{s} \boldsymbol{r} \times \boldsymbol{f}+\boldsymbol{m}_{\text {ext }}=\partial_{t}\left(\rho_{0} \hat{\boldsymbol{J}} \cdot \boldsymbol{\omega}\right)
\end{gathered}
$$

which has to be satisfied by the spatial stress resultants $\boldsymbol{f}=\hat{\boldsymbol{R}} \cdot \boldsymbol{F}$ and stress couples $\boldsymbol{m}=\hat{\boldsymbol{R}} \cdot \boldsymbol{M}$ with appropriate boundary conditions (see [10]). The inertial terms appearing on the r.h.s. of the equations of the balance of forces (linear momentum) Eqn. (4) and the balance of moments (angular momentum) Eqn. (5) depend parametrically on the local mass density $\rho_{0}(s)$ along the rod as well as on geometrical parameters of the local cross section (i.e.: area $A(s)$ and area moment tensor $\left.\hat{\boldsymbol{J}}(s, t)=\hat{\boldsymbol{R}} \cdot \hat{\boldsymbol{J}}_{0}(s) \cdot \hat{\boldsymbol{R}}^{T}\right)$ and contain the accelerations of the centerline positions $\partial_{t}^{2} \boldsymbol{r}(s, t)$ as well as the angular velocity vector $\boldsymbol{\omega}(s, t)$, which is implicitely defined by the temporal evolution equations $\partial_{t} \boldsymbol{a}^{(k)}=\boldsymbol{\omega} \times \boldsymbol{a}^{(k)}$ of the frame in close analogy to the Darboux vector, and its time derivative $\partial_{t} \boldsymbol{\omega}(s, t)$ as dynamical variables (see [10], [11] and [3] for details).

\subsection{Identification of minimal set of parameters for experimental work}

For slow motions like opening or closing doors and lids a quasistatic approach yields the best tradeof between accuracy and speed. In this approach we utilize a geometrically non-linear Cosserat rod model and combine it with a linear constitutive model [12] and [5]. When searching for a solution of the non-linear balance equations, we use the minimization of potential energy, which (besides gravity and frictionless contact interaction) is determined by the elastic potential energy

$$
\mathscr{V}=\frac{1}{2} \int_{0}^{L} \boldsymbol{\Gamma}^{T} \hat{\mathbb{C}}_{\Gamma} \boldsymbol{\Gamma}+\boldsymbol{K}^{T} \hat{\mathbb{C}}_{K} \boldsymbol{K} d s
$$

with $\hat{\mathbb{C}}_{\Gamma}=\operatorname{diag}\left(G A_{1}, G A_{2}, E A\right)$ and $\hat{\mathbb{C}}_{K}=\operatorname{diag}\left(E I_{1}, E I_{2}, G J_{T}\right)$. Here, the material strain and curvature measures are denoted as $\boldsymbol{\Gamma}$ and $\boldsymbol{K}$, respectively and the effective stiffness parameters of the cables

$$
\left[G A_{1}\right],\left[G A_{2}\right],[E A],\left[E I_{1}\right],\left[E I_{2}\right] \text { and }\left[G J_{T}\right]
$$

need to be determined for practical applications in cable simulation. For cables and hoses, we determine these parameters using the MeSOMICS test rig, a highly automated measurement setup that has been developed at ITWM [13] specially for cable simulation applications.

The kinematics of suspension linkage will, for instance, undergo substantially faster motions in dynamic operation scenarios in the frequency range up to $50 \mathrm{~Hz}$. As a consequence, inertial and damping effects play a considerable role in the motion of cables and hoses that are attached to these parts of the vehicle. In order to simulate these kinds of scenarios, we have to move from the quasistatic to a transient simulation approach. Here we assume a Kelvin-Voigt-type material to take damping effects into account [3], as the considered frequency range is still on the slow side, compared to much faster internal relaxation effects of the involved materials. Therefore, we additionally consider dissipative effects modeled by the so called dissipation function [14] ${ }^{1}$

$$
\mathscr{D}=\int_{0}^{L} \dot{\boldsymbol{\Gamma}}^{T} \hat{\mathbb{V}}_{\Gamma} \dot{\boldsymbol{\Gamma}}+\dot{\boldsymbol{K}}^{T} \hat{\mathbb{V}}_{K} \dot{\boldsymbol{K}} d s
$$

with $\hat{\mathbb{V}}_{\Gamma}=\operatorname{diag}\left(\eta_{G} A_{1}, \eta_{G} A_{2}, \eta_{E} A\right)$ and $\hat{\mathbb{V}}_{K}=\operatorname{diag}\left(\eta_{E} I_{1}, \eta_{E} I_{1}, \eta_{G} J_{T}\right)$, and driven by the strain and curvature rates $\dot{\Gamma}$ and $\dot{K}$. In addition to the above mentioned effective stiffness parameters we need to obtain the effective viscous parameters:

$$
\left[\eta_{G} A_{1}\right],\left[\eta_{G} A_{2}\right],\left[\eta_{E} A\right],\left[\eta_{E} I_{1}\right],\left[\eta_{E} I_{2}\right] \text { and }\left[\eta_{G} J_{T}\right] .
$$

\footnotetext{
${ }^{1}$ Eqn. (8) is deduced from the volumetric continuum version of the dissipation function of a Kelvin-Voigt solid, as discussed in [6] Ch. V \$34 and [7], adapted to our Cosserat rod in [3]. For details, see also [5].
} 
The number of parameters that need to be determined experimentally can be further reduced by utilizing theoretically deduced critical damping values for tensile, torsional and transverse shear vibrations [4]. In particular for tensile and shearing excitations, this is a reasonable simplification, since these have been shown to have negligible effects on the overall deformation behavior of the rod due to their high frequency and low amplitude characteristic.

According to the results of a linearized structural vibration analysis [4], effective critical damping values for extensional, torsional and transverse shear vibration modes are given by

$$
\left[\eta_{E} A\right]_{c r}=\frac{4 L}{\pi} \sqrt{\rho_{L}[E A]},\left[\eta_{G} J_{T}\right]_{c r}=\frac{4 L}{\pi} \sqrt{\left[\rho_{0} J_{T}\right]\left[G J_{T}\right]}, \quad\left[\eta_{G} A\right]_{c r}=2 \sqrt{\left[\rho_{0} I\right][G A]},
$$

where $\rho_{L}=\rho_{0} A$ is mass per length, and $\left[\rho_{0} I\right]=\rho_{L}(I / A)$ and $\left[\rho_{0} J_{T}\right]=2\left[\rho_{0} I\right]$ denote the rotational inertia parameters of the circular cross section, with $I_{1,2} \equiv I$ and $J_{T}=2 I$. While $\left[\eta_{E} A\right]_{c r}$ and $\left[\eta_{G} J_{T}\right]_{c r}$ scale proportional to the rod length $L,\left[\eta_{G} A\right]_{c r}$ scales proportional to the radius of gyration $\sqrt{I / A}$ of the cross section, independent of $L$.

For composite cables, neither $[G A]$ nor $\left[\eta_{G} A\right]$ are practically measurable quantities. Similarily, quasistatic and dynamic uniaxial tension experiments to determine $[E A]$ and $\left[\eta_{E} A\right]$ are plagued by systematic problems induced by often not well defined clamping of the cables at the boundaries. Although the approximation $[E A] \approx(A / I)[E I]$ typically underestimates tensional stiffness, it yields reasonable values for practical applications, as $[G A] \approx[E A] / 3$ does. Eqn. (10) may then be used to calibrate effective damping parameters $\left[\eta_{E} A\right]$ and $\left[\eta_{G} A\right]$ w.r.t. their critical values.

Differently, effective torsional damping is directly accessible via a simple experimental setup. Also bending oscillations of cables can be investigated experimentally, as demonstrated in [15].

\section{EXPERIMENT}

Two experimental prototypes (similar to the stiffness measurements) have been set up and are being tested at ITWM. One is a dynamic bending test (see Figs. 4 and 5), the other setup is a dynamic torsion test, following the standard torsional pendulum test for measuring the torsional stiffness of plastics [16] (see Fig. 6, 7 and 8). In both tests the specimens are fixed on one end. The free end is deflected mechanically. After releasing, the decaying free oscillation is recorded using a high speed camera.

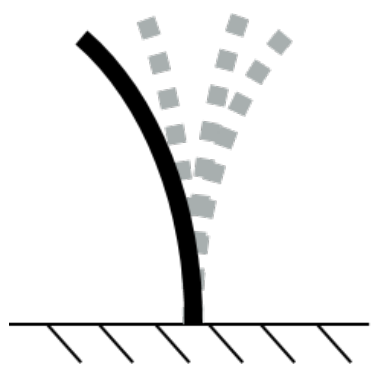

Figure 4: Schematic sketch of the experimental setup for bending oscillation measurements.

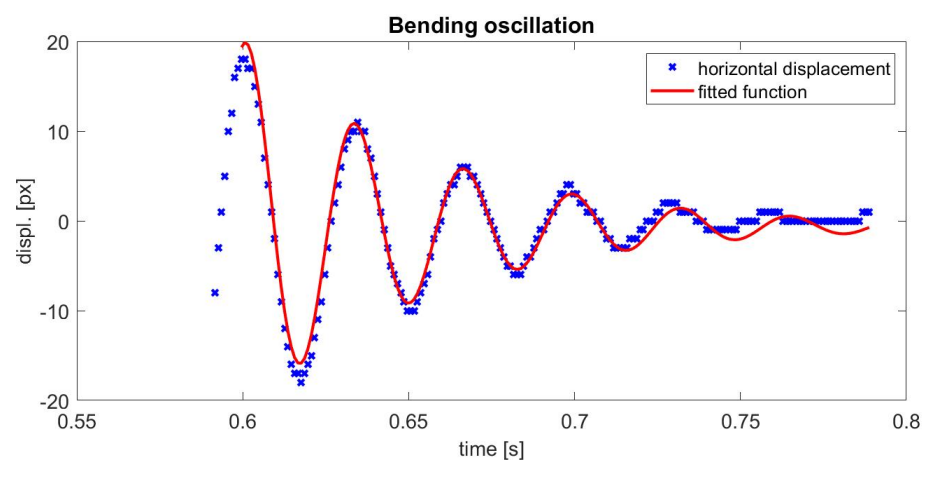

Figure 5: Data from an example measurement.

\subsection{Dynamic bending test}

The evaluation of the recorded data uses the lsqnonlin-function built-in in MATLAB to estimate the damped Eigenfrequency $\Omega$ and decay constant $\delta$ from the recorded data. Related dynamic bending stiffness and effective damping constant are computed based on Euler-Bernoulli beam theory

$$
[E I]_{d y n}=\omega^{2} C_{b c}, \quad\left[\eta_{E} I\right]=2 \delta C_{b c},
$$

with $\omega=\sqrt{\Omega^{2}+\delta^{2}}$ and constant $C_{b c}=\rho A\left(\frac{L}{\mu_{0}}\right)^{4}$ determined by boundary conditions. 


\subsection{Dynamic torsion test}

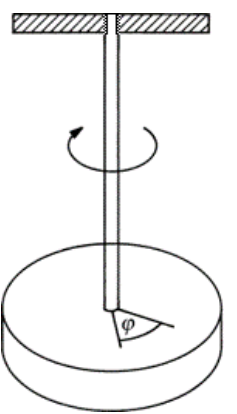

Figure 6: Measurement scheme for torsional oscillations.

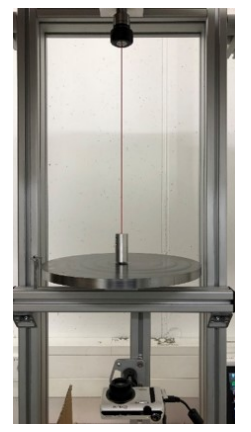

Figure 7: Experimental setup for torsion oscillations.

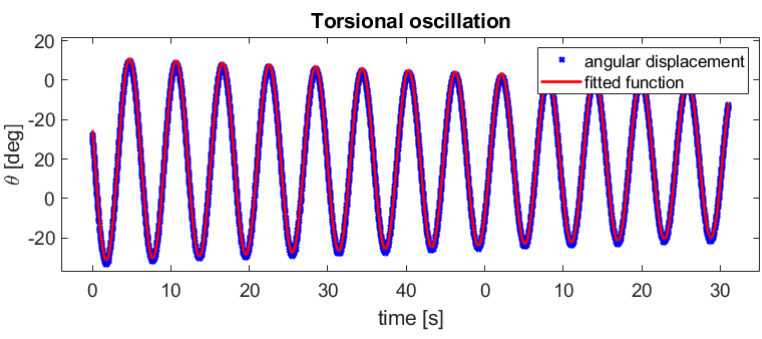

Figure 8: Extracted data from an example measurement.

The setup for measuring torsional oscillations (see Figs. 6, 7) resembles the well-known torsional pendulum, where the sample is fixed on one end, while the other end is attached to a heavy disc. After a manually induced initial torsional deflection, the experimental data displayed in Fig. 8 show that the disc rotates in a damped harmonic rotational oscillation.
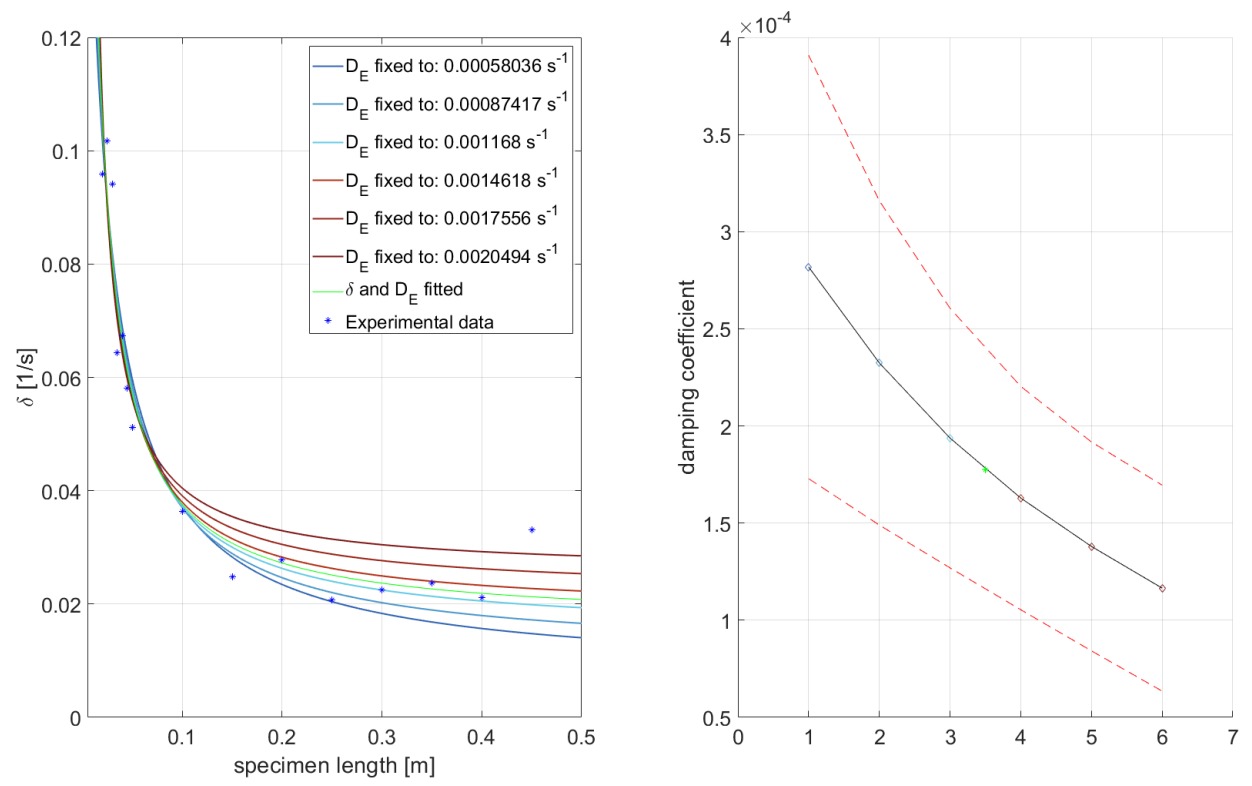

Figure 9: Torsional damping behavior for different specimen lengths. Left graph shows the $95 \%$ confidence interval for the fitting parameter related to $D_{E}$, depicted in six steps. Right graph shows the resulting uncertainty in $\delta$ for fixed values of $D_{E}$, based on the $95 \%$ confidence intervals of each individual fit with fixed values for $D_{E}$.

The recorded trajectories are fitted with a circular function to obtain the torsion angle $\varphi$ over time. The extracted data is then fitted analogous to the bending damping evaluation. With $J_{\text {Disc }}$ the inertial moment of the disc on the torsional pendulum and the cable mass neglected it holds

$$
[G J]_{d y n}=\omega^{2} L J_{D i s c}, \quad\left[\eta_{G} J\right] \approx 2 \delta L J_{\text {Disc }}-L D_{E},
$$

with $\omega=\sqrt{\Omega^{2}+\delta^{2}}$ and $D_{E}$ summarizing damping effects occurring at the fixation of the specimen. In order to determine $D_{E}$ we need to increase sample length as much as possible, since the 
term for the effective damping constant in (12) translates to

$$
\delta \approx \frac{\frac{\left[\eta_{G} J\right]}{L}+D_{E}}{2 J_{\text {Disc }}} \Rightarrow \delta \stackrel{L \rightarrow \infty}{\rightarrow} \frac{D_{E}}{2 J_{\text {Disc }}} .
$$

However, since space in our setup is limited and recorded data shows significant variance (see Fig. 9), the estimation of $D_{E}$ is still under investigation. Upon variation of the specimen length we expect a behavior of $\delta$ that follows a $\frac{1}{L}$-trajectory, see Eqn. (13). At the moment, the uncertainty of $D_{E}$ based on the $95 \%$ confidence interval in the fit considering $\left[\eta_{G} J\right]$ and $D_{E}$ as a parameter leads to a combined uncertainty range of about $184 \%$ for $\left[\eta_{G} J\right]$, spanning from $0.635 \times 10^{-4} s^{-1}$ (lowest point of the lower red dashed line depicted in the right graph of Fig. 9) to $3.908 \times 10^{-4} s^{-1}$ (highest point of the upper red dashed line depicted in the right graph of Fig. 9). This leads to a temporary loss of reliability for the results for $\left[\eta_{G} J\right]$. For further reading on the torsional damping measurement setup, see [17].

\section{APPLICATION IN SIMULATION}

We performed simulations of a cable of fixed length $(200 \mathrm{~mm})$ clamped to a rigid body that undergoes a sweep motion excitation (See Fig. 10).
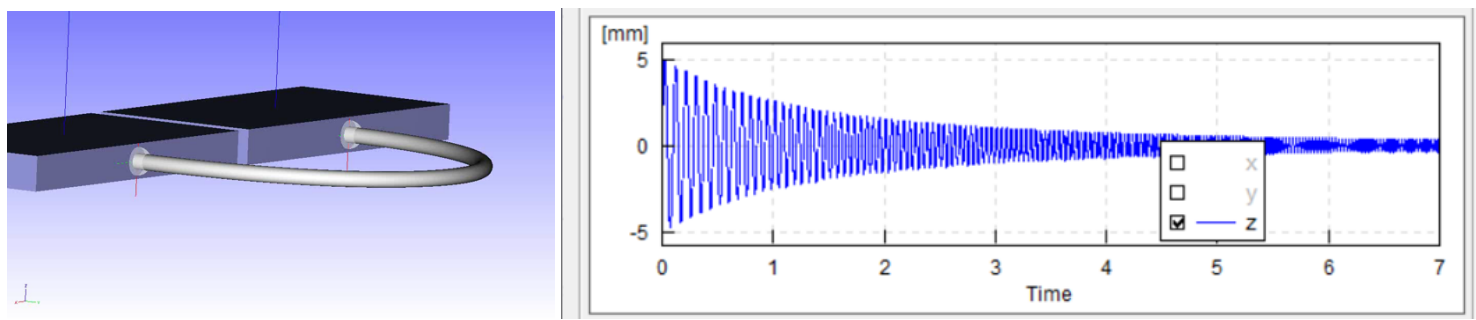

Figure 10: Setup of the virtual experiment (left) and applied sweep motion from $10 \mathrm{~Hz}$ to $38 \mathrm{~Hz}$ (right).

The resulting movement of the simulated cable is read out at the apex of the arch. In order to get a grasp of the sensitivity of a realistic use case against deviations in the measured damping value, we performed the simulation with varying values for $\left[\eta_{E} I\right]$. Reasonable boundaries for measured parameters are $\pm 50 \%$ of the measured value as an already conservative estimation. The results for this interval is shown in Fig. 11. It appears, that with decreasing damping, the size of the amplitude envelope increases as expected (from left to right in the graphics row).

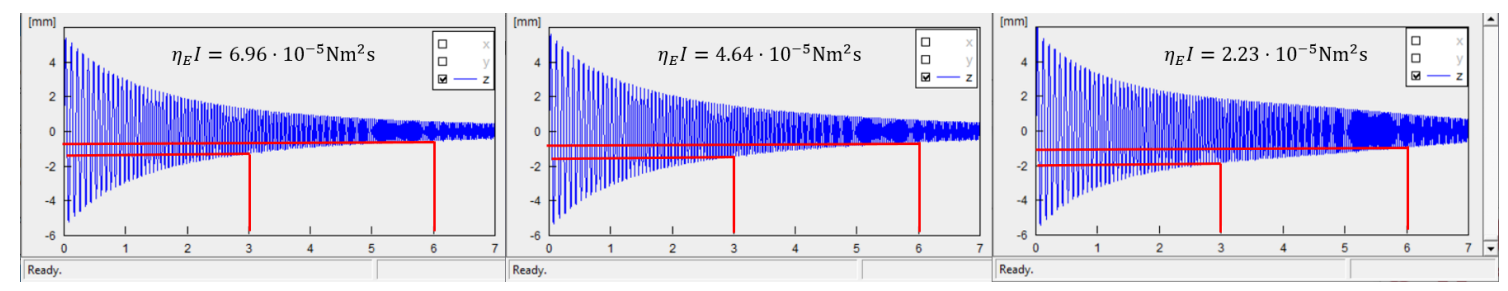

Figure 11: Resulting cable motion with values of 1.5 times the measured value, measured value and 0.5 times the measured value applied.

However, the changes in resulting motion are relatively small compared to the change in damping value. As a reference we compare the amplitudes at $3 \mathrm{~s}$ and $6 \mathrm{~s}$ of the sweep motion excitation, which refers to excitation frequencies of $22 \mathrm{~Hz}$ and $34 \mathrm{~Hz}$, respectively (see red lines in Fig. 11). The amplitudes at these points differ only within $\pm 1 \mathrm{~mm}$ from each other. In our field of application this represents a satisfying precision. 
However, since the behavior does not change its characteristic within these boundaries, we decreased the applied damping value further to $1 / 5$ th and 1/10th of the measured value respectively. The results are shown in Fig. 12, which starts with the measured value applied and shows the effect of decreasing damping value from left to right. This simulation shows a clear appearance of a resonance at $5.5 \mathrm{~s}$, which translates to $32 \mathrm{~Hz}$ in the sweep motion.

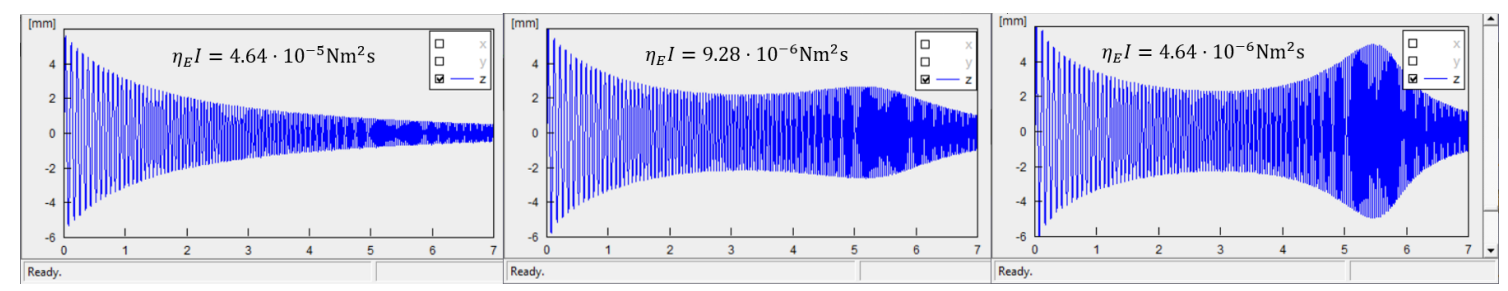

Figure 12: Resulting cable motion with values of measured value, $1 / 5$ th of the measured value and $1 / 10$ th of the measured value applied.

\section{CONCLUSION}

Our preliminary results are promising and indicate that the Kelvin-Voigt-type material model is suited to simulate dynamic behavior of cables and hoses within the considered time scale of low oscillation frequencies. Fitting the extracted motion data yields a damping value $\left[\eta_{E} I\right]$ (or $\left[\eta_{G} J\right]$ ) as well as a dynamic stiffness parameter $[E I]_{d y n}\left(\right.$ or $[G J]_{d y n}$ ). The obtained parameters can be used as effective mechanical properties within the framework of our simulation software.

While the results for bending oscillations have shown to be sufficiently robust, the interpretation of torsional oscillations is subject of ongoing research.

\section{OUTLOOK}

The simulation study shown in section 4 provides a lot of insight to the realistic use-case behavior. However, an additional validation experiment is subject of ongoing research in our department (see Fig. 13). This will yield further insight in terms of parametrization and applicability of the Kelvin-Voigt damping behavior.

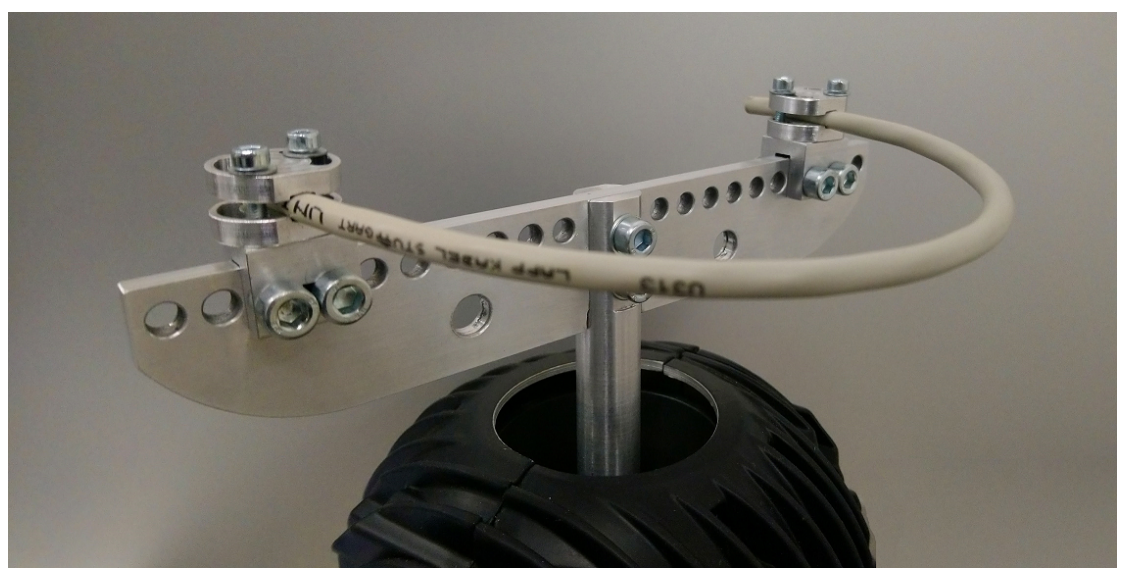

Figure 13: Experimental setup for validation of simulation results. 


\section{REFERENCES}

[1] R.M. Christensen, Theory of Viscoelasticity, p. 1-34, 1982.

[2] Olivier A. Bauchau and Nishant Nemani, Modeling viscoelastic behavior in flexible multibody systems, Multibody System Dynamics, 51(2), p. 159-194, 2020.

[3] H. Lang, J. Linn and M. Arnold, Multibody dynamics simulation of geometrically exact Cosserat rods, Multibody System Dynamics, 25(3), p. 285-312, 2011.

[4] H. Lang, S. Leyendecker and J. Linn, Numerical experiments for viscoelastic Cosserat rods with Kelvin-Voigt damping, Proceedings of ECCOMAS Thematic Conference on Multibody Dynamics, p. 453-462, Zagreb, Croatia, 2013.

[5] Linn, J. and Lang, H. and Tuganov, A., Geometrically exact Cosserat rods with Kelvin-Voigt type viscous damping, Mechanical Sciences, 4(1), p. 79-96, 2013.

[6] L. D. Landau and J. M. Lifshitz, Theory of Elasticity, 1986.

[7] Jean Lemaitre and Jean-Louis Chaboche, Mechanics of Solid Materials, 1990.

[8] Stuart S. Antman, Invariant Dissipative Mechanisms for the Spatial Motion of Rods Suggested by Artificial Viscosity, Journal of Elasticity, 70(1-3), p. 55-64, 2003.

[9] E. Reissner, On One-Dimensional Large-Displacement Finite-Strain Beam Theory, Studies in Applied Mathematics, 52(2), p. 87-95, 1973.

[10] J.C. Simo, A finite strain beam formulation. The three-dimensional dynamic problem. Part I, Computer Methods in Applied Mechanics and Engineering, 49(1), p. 55-70, 1985.

[11] Stuart S. Antman, Elasticity, Nonlinear Problems of Elasticity, p. 457-530, 1995.

[12] J. Linn, T. Hermannsson, F. Andersson and F. Schneider, Kinetic aspects of discrete Cosserat rods based on the difference geometry of framed curves, Proceedings of ECCOMAS Thematic Conference on Multibody Dynamics, p. 163-176, Prague, Czech Republic, 2017.

[13] MeSOMICS Homepage: www.mesomics.eu.

[14] L. D. Landau and J. M. Lifshitz, Statistical Physics - Part I, 1980.

[15] F. Schneider, Y. Kunz, J. Linn, V. Dörlich and F. Andersson, Kelvin-Voigt Damping Parameters for cosserat rod dynamics, ECCOMAS Multibody Dynamics Conference, Duisburg, Germany, 2019.

[16] Kunststoffe - Bestimmung dynamisch-mechanischer Eigenschaften - Teil 2: TorsionspendelVerfahren, DIN EN ISO 6721-2:2019-09.

[17] Philipp Knaus, Bestimmung der Torsionsdämpfung von Stäben und Kabeln, Bachelor's thesis, 2021, Technical University Kaiserslautern. 